Tôhoku Math. Journ.

24 (1972), 55-69.

\title{
ON POSITIVE CONVOLUTION OPERATORS FOR JACOBI SERIES
}

\author{
HERMAN BAVINCK
}

(Received Aug. 31, 1971)

\section{Introduction.}

1.1. In a preceding paper [2] the author has started the study of approximation of functions by processes, which are generated by the use of summability methods for the expansion of the functions in terms of Jacobi polynomials. The summability methods can be interpreted as convolution operators, if the convolution structure for Jacobi series, defined by Askey and Wainger [1], is used. By means of some general theorems on approximation processes on Banach spaces, (Berens [3]), it is possible to characterize the saturation class and the classes of non-optimal approximation of a number of classical summability methods for the summation of the Fourier-Jacobi series. This paper deals with saturation of positive convolution operators and the main part is a theorem of the Tureckii [10]-DeVore [4] type, which determines the saturation order and the saturation class of a sequence of positive convolution operators, satisfying a special condition on the Fourier-Jacobi coefficients of the kernel. The proof is a straight-forward generalization of DeVore's proof in the case of Fourier series. As applications, the saturation class of the higher order Jackson kernel and some other positive kernels are characterized.

1.2. We introduce some Banach spaces of complex valued functions on the interval $[-1,1]$. We write $C$ for the space of continuous functions, $L^{\infty}$ denotes the space of essentially bounded functions and we define the $L^{p}$ spaces with respect to the weight function $(x=\cos \theta)$

$$
\rho^{(\alpha, \beta)}(\theta)=\left(\sin \frac{\theta}{2}\right)^{2 \alpha+1}\left(\cos \frac{\theta}{2}\right)^{2 \beta+1} \quad\left(\alpha \geq \beta \geq-\frac{1}{2}\right) .
$$

We call $M$ the space of all regular finite Borel measures on $[-1,1]$. The spaces $C, L^{p}(1 \leq p \leq \infty)$ and $M$ are Banach spaces if endowed with the following norms 


$$
\begin{aligned}
& \|f\|_{c}=\sup _{0 \leqq \theta \leqq \pi}|f(\cos \theta)| \\
& \|f\|_{p}=\left[\int_{0}^{\pi}|f(\cos \theta)|^{p} \rho^{(\alpha, \beta)}(\theta) d \theta\right]^{1 / p} \quad(1 \leq p<\infty) \\
& \|f\|_{\infty}=\operatorname{ess}_{0 \leqq \theta \leqq \pi} \sup |f(\cos \theta)| \\
& \|\mu\|_{M}=\int_{0}^{\pi}|d \mu(\cos \theta)|
\end{aligned}
$$

With elements of these Banach spaces we can associate an expansion in terms of Jacobi polynomials. If $P_{n}^{(\alpha, \beta)}(x)$ is written for the Jacobi polynomial of degree $n$ and order $(\alpha, \beta)$ (see Szegö [9]), the functions

$$
R_{n}^{(\alpha, \beta)}(\cos \theta)=\frac{P_{n}^{(\alpha, \beta)}(\cos \theta)}{P_{n}^{(\alpha, \beta)}(1)}
$$

satisfy

$$
\int_{0}^{\pi} R_{n}^{(\alpha, \beta)}(\cos \theta) R_{m}^{(\alpha, \beta)}(\cos \theta) \rho^{(\alpha, \beta)}(\theta) d \theta=\delta_{n, m}\left[\omega_{n}^{(\alpha, \beta)}\right]^{-1}
$$

Here,

$$
\omega_{n}^{(\alpha, \beta)}=\frac{(2 n+\alpha+\beta+1) \Gamma(n+\alpha+\beta+1) \Gamma(n+\alpha+1)}{\Gamma(n+\beta+1) \Gamma(n+1) \Gamma(\alpha+1) \Gamma(\alpha+1)}=O\left(n^{2 \alpha+1}\right)(n \rightarrow \infty) .
$$

With $f$ belonging to one of the spaces $C$ or $L^{p}(1 \leq p \leq \infty)$ we associate the Fourier-Jacobi expansion

$$
f(\cos \theta) \sim \sum_{n=0}^{\infty} f^{\wedge}(n) \omega_{n}^{(\alpha, \beta)} R_{n}^{(\alpha, \beta)}(\cos \theta),
$$

where

$$
f^{\wedge}(n)=\int_{0}^{\pi} f(\cos \theta) R_{n}^{(\alpha, \beta)}(\cos \theta) \rho^{(\alpha, \beta)}(\theta) d \theta \quad(n=0,1, \cdots) .
$$

With a measure $\mu \in M$ we associate the Jacobi-Stieltjes expansion

$$
\mathrm{d} \mu(\cos \theta) \sim \sum_{n=0}^{\infty} \mu^{\vee}(n) \omega_{n}^{(\alpha, \beta)} R_{n}^{(\alpha, \beta)}(\cos \theta)
$$

where

$$
\mu^{\vee}(n)=\int_{0}^{\pi} R_{n}^{(\alpha, \beta)}(\cos \theta) d \mu(\cos \theta) \quad(n=0,1, \cdots) .
$$

Askey and Wainger [1] have introduced a generalized translation operator $T_{\phi}$, which maps a function $f$ with (1.4) into

$$
T_{\phi} f(\cos \theta) \sim \sum_{n=0}^{\infty} f^{\wedge}(n) \omega_{n}^{(\alpha, \beta)} R_{n}^{(\alpha, \beta)}(\cos \theta) R_{n}^{(\alpha, \beta)}(\cos \phi),
$$


and Gasper [5] has shown the positivity of this operator. This implies that $T_{\phi}$ has an operator norm 1 . If $f_{1}, f_{2} \in L^{1}$, then the convolution $f_{1} * f_{2}$ is defined by

$$
\left(f_{1} * f_{2}\right)(\cos \theta)=\int_{0}^{\pi} T_{\phi} f_{1}(\cos \theta) f_{2}(\cos \phi) \rho^{(\alpha, \beta)}(\phi) d \phi .
$$

This convolution has the usual properties (see Gasper [5]). If $f \in L^{p}$ $(1 \leq p \leq \infty)$ and $\mu \in M$ we can define the convolution $f * d \mu$ by

$$
(f * d \mu)(\cos \theta)=\int_{0}^{\pi} T_{\phi} f(\cos \theta) d \mu(\cos \phi) .
$$

Moreover, $f * d \mu \in L^{p}$ and the following inequality holds

$$
\|f * d \mu\|_{p} \leq\|f\|_{p}\|\mu\|_{M} \text {. }
$$

1.3. In the rest of this paper $X$ is written for one of the spaces $C$ or $L^{p}(1 \leq p<\infty)$. Assume that we are given a sequence $\left\{L_{n}\right\}$ of positive convolution operators, that is, $L_{n}$ has the form

$$
L_{n}(f ; \cos \theta)=\left(f * d \mu_{n}\right)(\cos \theta)=\int_{0}^{\pi} T_{\phi} f(\cos \theta) d \mu_{n}(\cos \phi) \quad(f \in X),
$$

where $\mu_{n}(n=1,2, \cdots)$ are non-negative elements of $M$ with

$$
\int_{0}^{\pi} d \mu_{n}(\cos \phi)=1 \text {. }
$$

We say that the sequence $\left\{L_{n}\right\}$ is saturated if there exists a nonincreasing sequence of positive numbers $\{\phi(n)\}$ with $\lim _{n \rightarrow \infty} \phi(n)=0$, such that

$$
\left\|f-L_{n}(f)\right\|_{X}=o(\phi(n))
$$$$
(n \rightarrow \infty)
$$

if and only if $f$ belongs to some "trivial" subspace of $X$ and

ii) there is a "non-trivial" element $f_{0} \in X$ satisfying

$$
\left\|f_{0}-L_{n}\left(f_{0}\right)\right\|_{X}=O(\phi(n)) \quad(n \rightarrow \infty) .
$$

The sequence $\{\phi(n)\}$ is then called the saturation order and the set $F(X$, $L_{n}$ ), which consists of all the elements of $X$ which satisfy ii), is called the saturation class or Favard class of $L_{n}$.

In this paper we shall prove a theorem, in which the behavior of the second trigonometric moment

$$
T\left(\mu_{n} ; 2\right)=\int_{0}^{\pi}\left(\sin \frac{\theta}{2}\right)^{2} d \mu_{n}(\cos \theta)
$$


determines the saturation of $\left\{L_{n}\right\}$. In section 2 we give some inequalities for Jacobi polynomials and we investigate the relationship between Jacobi coefficients and trigonometric moments. Then, following DeVore [4], we introduce the following conditions:

A. There exists a constant $C_{A}>0$ such that for each integer $k$ there is an $N(k)$ for which

$$
\frac{1-\mu_{n}^{\vee}(k)}{1-\mu_{n}^{\vee}(1)} \geq C_{A} k(k+\alpha+\beta+1) \quad \text { for } n>N(k) .
$$

B. There exists a constant $C_{B}>0$ such that for each $\varepsilon>0$ there is an $N(\varepsilon)$ such that

$$
\int_{0}^{\varepsilon}\left(\sin \frac{\theta}{2}\right)^{2} d \mu_{n}(\cos \theta) \geqq C_{B} \int_{0}^{\pi}\left(\sin \frac{\theta}{2}\right)^{2} d \mu_{n}(\cos \theta) \text { for } n>N(\varepsilon) .
$$

In section 3 we shall prove

1.4. Lemma. The conditions $\mathrm{A}$ and $\mathrm{B}$ are equivalent.

We define the Lipschitz classes with respect to the generalized translation operator by

$$
\operatorname{Lip}(\gamma, X)=\left\{f \in X: \exists c>0, \sup _{0<\psi<\phi}\left\|T_{\psi} f-f\right\|_{X} \leqq c \phi^{r}\right\}, \quad(0<\gamma \leq 2) .
$$

We now state the following theorem that will be proved in section 4 .

1.5. THEOREM. If $\left\{L_{n}\right\}$ is a sequence of operators of the form (1.12) and if either condition $A$ or condition $B$ is satisfied, then $\left\{L_{n}\right\}$ is saturated with order $\left(1-\mu_{n}^{\vee}(1)\right)$ and the saturation class $F\left(X, L_{n}\right)$ is $\operatorname{Lip}(2, X)$.

The Jacobi polynomials $R_{n}^{(\alpha, \beta)}(\cos \theta)$ satisfy the following differential equation:

(1.5) $-\frac{1}{\rho^{(\alpha, \beta)}(\theta)} \frac{d}{d \theta}\left\{\rho^{(\alpha, \beta)}(\theta) \frac{d}{d \theta} R_{n}^{(\alpha, \beta)}(\cos \theta)\right\}=n(n+\alpha+\beta+1) R_{n}^{(\alpha, \beta)}(\cos \theta)$.

If for $f \in X$ with the expansion (1.4) there exists an element $A f \in X$ such that

$$
A f \sim \sum_{n=0}^{\infty} n(n+\alpha+\beta+1) f^{\wedge}(n) \omega_{n}^{(\alpha, \beta)} R_{n}^{(\alpha, \beta)}(\cos \theta),
$$

then we say that $f \in D(A)$ and we call $A$ the operator which maps $D(A)$ into $X$ by $f \rightarrow A f$. The operator is the realization in $X$ of the differential operator

$$
-\frac{1}{\rho^{(\alpha, \beta)}(\theta)} \frac{d}{d \theta}\left\{\rho^{(\alpha, \beta)}(\theta) \frac{d}{d \theta}\right\}
$$


with boundary conditions $d / d \theta=0$ at $\theta=0$ and $\pi$, as follows from (1.15). Löfström and Peetre [7] have shown the close connection between the generalized translation operator $T_{\phi}$ and the operator $A$. In fact, for $f \in D(A)$ the following relations hold:

$$
\begin{gathered}
\left\|T_{\phi} f-f\right\|_{X} \leq C_{1}(\phi)\|A f\|_{X}, \\
\lim _{\phi \rightarrow 0+}\left\|\frac{f-T_{\phi} f}{C_{1}(\phi)}-A f\right\|_{X}=0,
\end{gathered}
$$

where

$$
C_{1}(\phi)=\int_{0}^{\phi} \frac{1}{\rho^{(\alpha, \beta)}(\theta)}\left(\int_{0}^{\theta} \rho^{(\alpha, \beta)}(\tau) d \tau\right) d \theta,
$$

(see Bavinck [2], section 4). Moreover,

$$
\lim _{\phi \rightarrow 0+} \frac{C_{1}(\phi)}{\sin ^{2} \frac{\phi}{2}}=\frac{1}{\alpha+1}
$$

and, since for $0<\phi \leq \pi / 2, \sqrt{2} / 2 \leq \cos \phi / 2 \leq 1$ we have

$$
\begin{array}{rlr}
C_{1}(\phi) & \leq \int_{0}^{\phi} \frac{1}{\rho^{(\alpha, \beta)}(\theta)}\left(\int_{0}^{\theta}\left(\sin \frac{\tau}{2}\right)^{2 \alpha+1} \cos \frac{\tau}{2} d \tau\right) d \theta & \\
& \leq \frac{1}{\alpha+1} \int_{0}^{\phi} \frac{\sin \frac{\theta}{2}}{\left(\cos \frac{\theta}{2}\right)^{2 \beta+1}} d \theta & \\
& \leq \frac{2^{\beta+1}}{\alpha+1} \sin ^{2} \frac{\phi}{2} & 0<\phi \leq \frac{\pi}{2} .
\end{array}
$$

Notation: We will use the notation $a_{n} \approx b_{n}(n \rightarrow \infty)$ if there are positive numbers $c_{1}$ and $c_{2}$ such that $c_{1} a_{n} \leq b_{n} \leq c_{2} a_{n}$.

\section{Some relations for Jacobi polynomials.}

2.1. Inequalities. We shall first prove the following inequalities for Jacobi polynomials $R_{k}^{(\alpha, \beta)}(x)$. Let $k$ be a natural number. Then

$$
1-R_{k}^{(\alpha, \beta)}(\cos \theta) \leq \frac{k(k+\alpha+\beta+1)}{\alpha+1} \sin ^{2} \frac{\theta}{2} \quad(0 \leq \theta \leq \pi) .
$$

There exists a constant $c_{\alpha}>0$, such that for $0<\varepsilon<4 /(2 k+\alpha+\beta+2)$

$$
c_{\alpha} \frac{k(k+\alpha+\beta+1)}{\alpha+1} \sin ^{2} \frac{\theta}{2} \leq 1-R_{k}^{(\alpha, \beta)}(\cos \theta) \quad(0 \leq \theta \leq \varepsilon) .
$$

By the differentiation formula 


$$
\frac{d}{d x} R_{k}^{(\alpha, \beta)}(x)=\frac{k(k+\alpha+\beta+1)}{2(\alpha+1)} R_{k-1}^{(\alpha+1, \beta+1)}(x)
$$

we obtain from the mean-value theorem

$$
1-R_{k}^{(\alpha, \beta)}(\cos \theta)=\frac{k(k+\alpha+\beta+1)}{(\alpha+1)} \sin ^{2} \frac{\theta}{2} R_{k-1}^{(\alpha+1, \beta+1)}(\cos \bar{\theta}), 0 \leq \bar{\theta} \leq \theta .
$$

Since $\left|R_{k-1}^{(\alpha+1, \beta+1)}(\cos \bar{\theta})\right| \leq 1,0 \leq \bar{\theta} \leq \pi$, formula (2.1) follows.

For the proof of (2.2) we use Hilb's formula (Szegö [9], $(8,21.12)$ ) for large $n$

$$
\begin{gathered}
\left(\sin \frac{\theta}{2}\right)^{\alpha}\left(\cos \frac{\theta}{2}\right)^{\beta} R_{n}^{(\alpha, \beta)}(\cos \theta)=N^{-\alpha} \Gamma(\alpha+1)(\theta / \sin \theta)^{1 / 2} J_{\alpha}(N \theta) \\
+ \begin{cases}\theta^{1 / 2} O\left(n^{-3 / 2-\alpha}\right), & \text { if } c n^{-1} \leq \theta \leq \pi-\varepsilon, \\
\theta^{\alpha+2} O(1), & \text { if } 0<\theta \leq c n^{-1},\end{cases}
\end{gathered}
$$

where $N=n+(\alpha+\beta+1) / 2$.

The power series expansion of $(z / 2)^{-\alpha} J_{\alpha}(z)$ has terms with alternating sign, and monotonically decreasing for real $z, 0<z<2$. Hence we have

$$
\begin{aligned}
R_{n-1}^{(\alpha+1, \beta+1)}(\cos \theta) & \geq \Gamma(a+2)\left(\frac{2}{N \theta}\right)^{\alpha+1} J_{\alpha+1}(N \theta)+\theta^{2} O(1) 0 \leq \theta<2 N^{-1} \\
& \geq 1-\frac{\left(\frac{N \theta}{2}\right)^{2}}{\alpha+2}+\theta^{2} O(1) \\
& >\frac{\alpha+1}{\alpha+2}-O\left(N^{-2}\right) .
\end{aligned}
$$

The inequality (2.2) follows from (2.3) and (2.4) for $k \geq k_{0}$. On the other hand, the constant $c_{\alpha}$ can be chosen in such a way, that (2.2) remains valid for $k \leq k_{0}$.

2.2. Relations between trigonometric moments and Jacobi coefficients. The following expansion is a simple consequence of Rodrigues' formula (see also Szegö [9], formula (9.3.11)).

$$
\begin{aligned}
& \left(\sin \frac{\theta}{2}\right)^{2 \sigma} \\
= & \frac{\Gamma(\sigma+1) \Gamma(\sigma+\alpha+1)}{\Gamma(\alpha+1)} \sum_{n=0}^{\sigma}(-1)^{n} \frac{(2 n+\alpha+\beta+1) \Gamma(n+\alpha+\beta+1)}{\Gamma(\sigma-n+1) \Gamma(n+\alpha+\beta+\sigma+2) \Gamma(n+1)} \\
& \times R_{n}^{(\alpha, \beta)}(\cos \theta) \quad(\sigma=1,2, \cdots) .
\end{aligned}
$$

From the expression of the Jacobi polynomials in terms of hypergeometric functions 


$$
R_{n}^{(\alpha, \beta)}(\cos \theta)={ }_{2} F_{1}\left(-n, n+\alpha+\beta+1 ; \alpha+1 ; \sin ^{2} \frac{\theta}{2}\right)
$$

we easily derive

(2.6) $1-R_{n}^{(\alpha, \beta)}(\cos \theta)$

$$
=\sum_{k=1}^{n}(-1)^{k+1} \frac{\Gamma(n+\alpha+\beta+k+1) \Gamma(n+1) \Gamma(\alpha+1)}{\Gamma(n-k+1) \Gamma(n+\alpha+\beta+1) \Gamma(k+\alpha+1) \Gamma(k+1)} \sin ^{2 k} \frac{\theta}{2} .
$$

If the trigonometric moment of order $2 \sigma(\sigma=1,2, \cdots)$ is defined by

$$
T\left(\mu_{n} ; 2 \sigma\right)=\int_{0}^{\pi}\left(\sin \frac{\theta}{2}\right)^{2 \sigma} d \mu_{n}(\cos \theta),
$$

we obtain by (2.5), noticing the value of (2.5) at $\theta=0$,

(2.7) $T\left(\mu_{n} ; 2 \sigma\right)$

$$
\begin{aligned}
= & \frac{\Gamma(\sigma+1) \Gamma(\sigma+\alpha+1)}{\Gamma(\alpha+1)} \sum_{k=1}^{\sigma}(-1)^{k+1} \frac{(2 k+\alpha+\beta+1) \Gamma(k+\alpha+\beta+1)}{\Gamma(\sigma-k+1) \Gamma(k+\alpha+\beta+\sigma+2) \Gamma(k+1)} \\
& \times\left(1-\mu_{n}^{\vee}(k)\right) .
\end{aligned}
$$

On the other hand (2.6) leads to

(2.8) $\quad 1-\mu_{n}^{\vee}(k)$

$$
=\frac{\Gamma(k+1) \Gamma(\alpha+1)}{\Gamma(k+\alpha+\beta+1)} \sum_{\sigma=1}^{k}(-1)^{\sigma+1} \frac{\Gamma(k+\alpha+\beta+\sigma+1)}{\Gamma(k-\sigma+1) \Gamma(\sigma+\alpha+1) \Gamma(\sigma+1)} T\left(\mu_{n} ; 2 \sigma\right) .
$$

Hence, we easily derive from (2.7)

$$
T\left(\mu_{n} ; 2\right)=\frac{\alpha+1}{\alpha+\beta+2}\left(1-\mu_{n}^{\vee}(1)\right)
$$

and

$$
\frac{T\left(\mu_{n} ; 4\right)}{T\left(\mu_{n} ; 2\right)}=\frac{(\alpha+2)(\alpha+\beta+2)}{(\alpha+\beta+3)(\alpha+\beta+4)}\left[\frac{2(\alpha+\beta+3)}{\alpha+\beta+2}-\frac{1-\mu_{n}^{\vee}(2)}{1-\mu_{n}^{\vee}(1)}\right] .
$$

From (2.8) and (2.9). we conclude

$$
\begin{aligned}
& \frac{1-\mu_{n}^{\vee}(k)}{1-\mu_{n}^{\vee}(1)} \\
= & \frac{k(k+\alpha+\beta+1)}{\alpha+\beta+2}-\frac{\Gamma(k+1) \Gamma(\alpha+2)}{(\alpha+\beta+2) \Gamma(k+\alpha+\beta+1)} \\
& \times \sum_{\sigma=2}^{k}(-1)^{\sigma} \frac{\Gamma(k+\alpha+\beta+\sigma+1)}{\Gamma(k-\sigma+1) \Gamma(\sigma+\alpha+1) \Gamma(\sigma+1)} \frac{T\left(\mu_{n} ; 2 \sigma\right)}{T\left(\mu_{n} ; 2\right)} .
\end{aligned}
$$

Similar relations between trigonometric moments and Fourier coefficients have been established by Stark [8]. We also have the following theorem, 
which generalizes a result of Görlich and Stark [6] (see also Stark [8]).

2.3. THEonem. For a sequence $\left\{L_{n}\right\}$ of positive convolution operators of the form (1.12) the following assertions are equivalent:

$$
\lim _{n \rightarrow \infty} \frac{1-\mu_{n}^{\vee}(k)}{1-\mu_{n}^{\vee}(1)}=\frac{k(k+\alpha+\beta+1)}{\alpha+\beta+2} \quad(k=1,2, \cdots) .
$$

$$
\lim _{n \rightarrow \infty} \frac{1-\mu_{n}^{\vee}(2)}{1-\mu_{n}^{\vee}(1)}=\frac{2(\alpha+\beta+3)}{\alpha+\beta+2},
$$

$$
\lim _{n \rightarrow \infty} \frac{T\left(\mu_{n} ; 4\right)}{T\left(\mu_{n} ; 2\right)}=0 \text {. }
$$

Proof. Relation (b) is a trivial consequence of (a). Relation (c) follows from (b) by (2.10). Since $0 \leq \sin ^{2} \theta / 2 \leq 1$ and the measures $\mu_{n}$ are positive it is obvious that

$$
T\left(\mu_{n} ; 2 \sigma\right) \leqq T\left(\mu_{n} ; 4\right) \quad \text { for } \sigma \geq 2 .
$$

Therefore relation (c) implies that $\lim _{n \rightarrow \infty} T\left(\mu_{n} ; 2 \sigma\right) / T\left(\mu_{n} ; 2\right)=0, \sigma \geq 2$. Thus, by formula (2.11) relation (a) follows.

3. Proof of lemma 1.4. We first show that $B$ implies $A$. If we take $\varepsilon<4 /(2 k+\alpha+\beta+2)$ and $N(\varepsilon)$ as given in $\mathrm{B}$, we have, using (2.2) and (2.9), for $n>N(\varepsilon)$

$$
\begin{aligned}
1-\mu_{n}^{\vee}(k) & =\int_{0}^{\pi}\left(1-R_{k}^{(\alpha, \beta)}(\cos \theta)\right) d \mu_{n}(\cos \theta) \\
& \geq \int_{0}^{\varepsilon}\left(1-R_{k}^{(\alpha, \beta)}(\cos \theta)\right) d \mu_{n}(\cos \theta) \\
& \geq c_{\alpha} \frac{k(k+\alpha+\beta+1)}{\alpha+1} \int_{0}^{\varepsilon} \sin ^{2} \frac{\theta}{2} d \mu_{n}(\cos \theta) \\
& \geq c_{\alpha} \frac{k(k+\alpha+\beta+1)}{\alpha+1} C_{B} \int_{0}^{\pi} \sin ^{2} \frac{\theta}{2} d \mu_{n}(\cos \theta) \\
& =\frac{c_{\alpha} C_{B}}{\alpha+\beta+2} k(k+\alpha+\beta+1)\left(1-\mu_{n}^{\vee}(1)\right) .
\end{aligned}
$$

Therefore, A holds with $N(k)=N(\varepsilon)$ and $C_{A}=\left(c_{\alpha} C_{B}\right) /(\alpha+\beta+2)$.

We will now show that $\mathrm{A}$ implies $\mathrm{B}$ with $C_{B}=C_{A}(\alpha+\beta+2) / 2$. We choose $\varepsilon=\varepsilon_{0}$ and we consider the measures

$$
\nu_{n}(\cos \theta)= \begin{cases}0, & 0 \leq \theta<\varepsilon_{0}, \\ \frac{1}{T\left(\mu_{n} ; 2\right)} \mu_{n}(\cos \theta), & \varepsilon_{0} \leq \theta \leq \pi .\end{cases}
$$

Then 


$$
\int_{0}^{\pi} d \nu_{n}(\cos \theta) \leq \frac{1}{\sin ^{2} \frac{\varepsilon_{0}}{2}} \frac{1}{T\left(\mu_{n} ; 2\right)} \int_{0}^{\pi} \sin ^{2} \frac{\theta}{2} d \mu_{n}(\cos \theta)=\frac{1}{\sin ^{2} \frac{\varepsilon_{0}}{2}} .
$$

Thus, we have for each $k(k=1,2, \cdots)$

$$
\int_{0}^{\pi}\left\{1-R_{k}^{(\alpha, \beta)}(\cos \theta)\right\} d \nu_{n}(\cos \theta) \leq \frac{2}{\sin ^{2} \frac{\varepsilon_{0}}{2}} .
$$

Choose $k_{0}$ so large that

$$
\frac{C_{A} k_{0}\left(k_{0}+\alpha+\beta+1\right)(\alpha+\beta+2)}{4(\alpha+1)} \geq \frac{1}{\sin ^{2} \frac{\varepsilon_{0}}{2}}
$$

Then,

$$
\begin{aligned}
& \frac{1}{T\left(\mu_{n} ; 2\right)} \int_{0}^{\varepsilon_{0}}\left\{1-R_{k_{0}}^{(\alpha, \beta)}(\cos \theta)\right\} d \mu_{n}(\cos \theta) \\
= & \frac{1}{T\left(\mu_{n} ; 2\right)} \int_{0}^{\pi}\left\{1-R_{k_{0}}^{(\alpha, \beta)}(\cos \theta)\right\} d \mu_{n}(\cos \theta) \\
& -\int_{0}^{\pi}\left\{1-R_{k_{0}}^{(\alpha, \beta)}(\cos \theta)\right\} d \nu_{n}(\cos \theta) \\
\geq & \frac{1}{T\left(\mu_{n} ; 2\right)} \int_{0}^{\pi}\left\{1-R_{k_{0}}^{(\alpha, \beta)}(\cos \theta)\right\} d \mu_{n}(\cos \theta) \\
& -\frac{C_{A} k_{0}\left(k_{0}+\alpha+\beta+1\right)(\alpha+\beta+2)}{2(\alpha+1)} .
\end{aligned}
$$

By virtue of condition A we have for $n \geq N\left(k_{0}\right)$

$$
\begin{aligned}
& \int_{0}^{\varepsilon_{0}}\left\{1-R_{k_{0}}^{(\alpha, \beta)}(\cos \theta)\right\} d \mu_{n}(\cos \theta) \\
\geq & C_{A} k_{0}\left(k_{0}+\alpha+\beta+1\right) \frac{(\alpha+\beta+2)}{2(\alpha+1)} \int_{0}^{\pi} \sin ^{2} \frac{\theta}{2} d \mu_{n}(\cos \theta) .
\end{aligned}
$$

Finally, by (2.1) we have

$$
\begin{aligned}
\int_{0}^{\varepsilon_{0}} \sin ^{2} \frac{\theta}{2} d \mu_{n}(\cos \theta) & \geq \frac{(\alpha+1)}{k_{0}\left(k_{0}+\alpha+\beta+1\right)} \int_{0}^{\varepsilon_{0}}\left\{1-R_{k_{0}}^{(\alpha, \beta)}(\cos \theta)\right\} d \mu_{n}(\cos \theta) \\
& \geq C_{A} \frac{(\alpha+\beta+2)}{2} \int_{0}^{\pi} \sin ^{2} \frac{\theta}{2} d \mu_{n}(\cos \theta)
\end{aligned}
$$

which proves lemma 1.4 . 
4. Proof of theorem 1.5. Let $\left\{L_{n}\right\}$ be a sequence of positive linear operators of the form (1.12) which satisfy either condition A or B. On account of lemma 1.4 both conditions $\mathrm{A}$ and $\mathrm{B}$ are satisfied and we will interchange them appropriately.

We first show that $\left\{L_{n}\right\}$ is saturated with order $\left(1-\mu_{n}^{\vee}(1)\right)$. If $f \in X$ and

$$
\left\|L_{n}(f)-f\right\|_{X}=o\left(1-\mu_{n}^{\vee}(1)\right) \quad(n \rightarrow \infty),
$$

then

$$
f^{\wedge}(k)-f^{\wedge}(k) \mu_{n}^{\vee}(k)=o\left(1-\mu_{n}^{\vee}(1)\right) \quad(n \rightarrow \infty) .
$$

In view of condition $\mathrm{A}$ this implies $f^{\wedge}(k)=0, k=1,2, \cdots$, and therefore $f$ is a constant. The function $f_{0}(\cos \theta)=(\sin \theta / 2)^{2}$ is an example of a non-constant function which satisfies

$$
\left\|L_{n}(f)-f\right\|_{X}=O\left(1-\mu_{n}^{\vee}(1)\right) \quad(n \rightarrow \infty) .
$$

Hence $\left\{L_{n}\right\}$ is saturated with order $\left(1-\mu_{n}^{\vee}(1)\right)$. The "trivial" subspace used in section 1.3 is here the space of constant functions.

We now wish to characterize the saturation class $F\left(X, L_{n}\right)$. An element $f \in X$ belongs to $F\left(X, L_{n}\right)$ if and only if

$$
\left\|\int_{0}^{\pi}\left(T_{\phi} f(\cos \theta)-f(\cos \theta)\right) d \mu_{n}(\cos \phi)\right\|_{X}=O\left(1-\mu_{n}^{\vee}(1)\right) \quad(n \rightarrow \infty),
$$

or equivalently

$$
\left\|\int_{0}^{\pi} \frac{\left(T_{\phi} f(\cos \theta)-f(\cos \theta)\right)}{\sin ^{2} \frac{\phi}{2}} d \psi_{n}(\phi)\right\|_{X}=O(1) \quad(n \rightarrow \infty),
$$

where

$$
d \psi_{n}(\phi)=\frac{(\alpha+\beta+2) \sin ^{2} \frac{\phi}{2} d \mu_{n}(\cos \phi)}{(\alpha+1)\left(1-\mu_{n}^{\vee}(1)\right)} .
$$

By (2.9) $\int_{0}^{\pi} d \psi_{n}(\phi)=1, n=1,2, \cdots$ and consequently it is clear that $f \in F\left(X, L_{n}\right)$, if $f \in \operatorname{Lip}(2, X)$ (see (1.14)).

We still have to prove that $f \in F\left(X, L_{n}\right)$ implies $f \in \operatorname{Lip}(2, X)$. If we denote by $A$ the operator defined by (1.14), then we will first show that for $f \in D(A)$ satisfying

$$
\left\|f-L_{n}(f)\right\|_{X} \leq M\left(1-\mu_{n}^{\vee}(1)\right)
$$$$
(n \rightarrow \infty),
$$

the following inequality is valid: 


$$
\|A f\|_{X} \leq C\left(M+\|f\|_{X}\right) .
$$

Here $C$ is a constant independent of $f$.

Since the measures $\psi_{n}$ all have norm 1 , there exists a subsequence $\left\{n_{j}\right\}$ and a measure $\psi$ such that $\left\{\psi_{n_{j}}\right\}$ converges weakly* to $\psi$. By condition $B$ and the weak* convergence it follows that for each $\varepsilon>0$

$$
\int_{0}^{\varepsilon} d \psi=\lim _{j \rightarrow \infty} \int_{0}^{\varepsilon} d \psi_{n_{j}} \geq C_{B} .
$$

We choose $\varepsilon_{0}$ so small that $\varepsilon_{0} \leq \pi / 2$ and

$$
\int_{\left(0, \varepsilon_{0}\right)} d \psi \leq \frac{C_{B}}{S} \quad \text { with } S>2+2^{\beta+2} \text {. }
$$

For $f \in D(A)$ satisfying (4.1) we have, putting $M^{\prime}=(\alpha+\beta+2) M /(\alpha+1)$,

$$
\left\|\int_{0}^{\pi} \frac{T_{\phi} f-f}{\sin ^{2} \frac{\phi}{2}} d \psi(\phi)\right\|_{x} \leq \lim _{j \rightarrow \infty}\left\|\int_{0}^{\pi} \frac{T_{\phi} f-f}{\sin ^{2} \frac{\phi}{2}} d \psi_{n_{j}}(\phi)\right\| \leq M^{\prime} .
$$

Hence,

$$
\begin{aligned}
\left\|\int_{0}^{\varepsilon_{0}} \frac{T_{\phi} f-f}{\sin ^{2} \frac{\phi}{2}} d \psi(\phi)\right\|_{X} & \leq M^{\prime}+\left\|\int_{\varepsilon_{0}}^{\pi} \frac{T_{\phi} f-f}{\sin ^{2} \frac{\phi}{2}} d \psi(\phi)\right\|_{X} \\
& \leq M^{\prime}+\frac{2\|f\|_{X}}{\sin ^{2} \frac{\varepsilon_{0}}{2}} .
\end{aligned}
$$

From (1.18) and (1.20) we know that $\left(T_{\phi} f-f\right) /\left(\sin ^{2}(\phi / 2)\right) \rightarrow-1 /(\alpha+1)$ $A f$ in $X$ if $\phi \rightarrow 0^{+}$. In virtue of (4.3) and (4.4)

$$
\begin{aligned}
& \left\|\int_{0}^{\varepsilon_{0}} \frac{T_{\phi} f-f}{\sin ^{2} \frac{\phi}{2}} d \psi(\phi)\right\|_{X} \\
\geq & \left(1-\frac{1}{S}\right) C_{B} \frac{1}{\alpha+1}\|A f\|_{X}-\left\|\int_{\left(0, \varepsilon_{0}\right)} \frac{T_{\phi} f-f}{\sin ^{2} \frac{\phi}{2}} d \psi(\phi)\right\|_{X} .
\end{aligned}
$$

Since by (1.17) and (1.21)

$$
\left\|\frac{T_{\phi} f-f}{\sin ^{2} \frac{\phi}{2}}\right\|_{X} \leq \frac{2^{\beta+1}}{\alpha+1}\|A f\|_{X}, \quad 0<\phi \leq \frac{\pi}{2},
$$

we derive from (4.6) and (4.4) 


$$
\begin{aligned}
& \left\|\int_{0}^{\varepsilon_{0}} \frac{T_{\phi} f-f}{\sin ^{2} \frac{\phi}{2}} d \psi(\phi)\right\|_{X} \\
\geq & \left(1-\frac{1}{S}\right) C_{B} \frac{1}{\alpha+1}\|A f\|_{X}-\frac{C_{B}}{S} \frac{2^{\beta+1}}{\alpha+1}\|A f\|_{\lambda} \\
\geq & \frac{1}{2(\alpha+1)} C_{B}\|A f\|_{X},
\end{aligned}
$$

as we have chosen $S>2+2^{\beta+2}$.

Hence (4.7) and (4.5) yield

$$
\|A f\|_{X} \leq \frac{2(\alpha+1)}{C_{B}}\left(M^{\prime}+\frac{2\|f\|_{X}}{\sin ^{2} \frac{\varepsilon_{0}}{2}}\right),
$$

which establishes (4.2), if $C$ is chosen appropriately.

If we take an arbitrary element of $F\left(X, L_{n}\right)$ such that

$$
\left\|f-L_{n}(f)\right\|_{X} \leq M\left(1-\mu_{n}^{\vee}(1)\right) \quad(n=1,2, \cdots),
$$

then we study the convolution of $f$ with a positive polynomial kernel $K_{m}$ (for instance the de la Vallée-Poussin kernel (see section (5.1)) $f_{m}=f * K_{m}$, which clearly belongs to $D(A)$. Then for $f_{m}$

$$
\begin{aligned}
\left\|f_{m}-L_{n}\left(f_{m}\right)\right\|_{X} & =\left\|f * K_{m}-f * K_{m} * d \mu_{n}\right\|_{x}=\left\|\left(f-f * d \mu_{n}\right) * K_{m}\right\|_{X} \\
& \leq\left\|f-f * d \mu_{n}\right\|_{X} \leq M\left(1-\mu_{n}^{\vee}(1)\right) \quad(n=1,2, \cdots) .
\end{aligned}
$$

Since $\left\|f_{m}\right\|_{X} \leqq\|f\|_{X}$ holds, it follows from (4.2) that

$$
\left\|A f_{m}\right\|_{X} \leq C\left(M+\left\|f_{m}\right\|_{X}\right) \leq C\left(M+\|f\|_{X}\right) \text {. }
$$

Hence for $\phi>0$ it follows from (1.17) and (1.21)

$$
\left\|\frac{T_{\phi} f_{m}-f_{m}}{\phi^{2}}\right\|_{X} \leq \frac{2^{\beta-1}}{\alpha+1}\left\|A f_{m}\right\|_{X} \leq C_{1}\left(M+\|f\|_{X}\right), \quad(m=1,2, \cdots) .
$$

If we take the limit as $m \rightarrow \infty$ in we get

$$
\left\|\frac{T_{\phi} f-f}{\phi^{2}}\right\|_{X} \leq C_{1}\left(M+\|f\|_{X}\right)
$$

which is equivalent with $f \in \operatorname{Lip}(2, X)$.

5. Applications. We will show in this section, that many of the classical approximation processes which have a positive kernel, satisfy the conditions of theorem 2.3. Since condition (a) of theorem 2.3 is essentially stronger than condition $\mathrm{A}$ of theorem 1.5, we may conclude by theorem 1.5, that these approximation processes are saturated with order 
$\left(1-\mu_{n}^{\vee}(1)\right)$ and that their saturation class in $\operatorname{Lip}(2, X)$. For some of the examples given here, these results have already been obtained by different methods in Bavinck [2].

5.1. The de la Vallée-Poussin summability process. The de la Vallée-Poussin kernel is defined by

$$
V_{N}(\cos \theta)=\omega_{0}^{(\alpha, \beta+N)}\left(\cos \frac{\theta}{2}\right)^{2 N} \quad N=1,2, \cdots,
$$

where $\omega_{0}^{(\alpha, \beta+N)}$ is given in (1.3). The trigonometric moments of $V_{N}$ are very easy to calculate:

$$
T\left(V_{N} ; 2 \sigma\right)=\frac{\omega_{0}^{(\alpha, \beta+N)}}{\omega_{0}^{(\alpha+\sigma, \beta+N)}} .
$$

Hence

$$
\lim _{N \rightarrow \infty} \frac{T\left(V_{N} ; 4\right)}{T\left(V_{N} ; 2\right)}=\lim _{N \rightarrow \infty} \frac{\omega_{0}^{(\alpha+1, \beta+N)}}{\omega_{0}^{(\alpha+2, \beta+N)}}=\lim _{N \rightarrow \infty} \frac{\alpha+2}{N+\alpha+\beta+3}=0 .
$$

By theorem 2.3 and theorem 1.5 we conclude that the summability process $V_{N} f(\cos \theta)=\left(f * V_{N}\right)(\cos \theta)$ is saturated with the order $1-V_{N}^{\wedge}(1)$, which by (2.9) is

$$
1-V_{N}^{\wedge}(1)=\frac{(\alpha+\beta+2)}{(\alpha+1)} T\left(V_{N} ; 2\right)=\frac{\alpha+\beta+2}{N+\alpha+\beta+2} .
$$

The saturation class is $\operatorname{Lip}(2, X)$.

5.2. The Jackson kernel. We now direct our attention to the Jackson kernel

$$
L_{n, r}(\theta)=\lambda_{n, r}^{-1}\left(\frac{\sin n \frac{\theta}{2}}{\sin \frac{\theta}{2}}\right)^{2 r} \quad \begin{aligned}
& (r \text { and } n \text { positive integer }, \\
& r>\alpha+2),
\end{aligned}
$$

where

$$
\lambda_{n, r}=\int_{0}^{\pi}\left(\frac{\sin n \frac{\theta}{2}}{\sin \frac{\theta}{2}}\right)^{2 r} \rho^{(\alpha, \beta)}(\theta) d \theta \approx n^{2 r-2 \alpha-2} .
$$

In order to find the saturation order and the saturation class, we show that the kernel (5.2) satisfies condition $B$ of theorem 1.4. Using the wellknown estimates $\theta / \pi \leq \sin \theta / 2 \leq \theta / 2$ for $0 \leq \theta \leq \pi$ and $\sqrt{2} / 2 \leq \cos \theta / 2 \leq 1$ for $0 \leq \theta \leq \pi / 2$ we have 


$$
\begin{aligned}
\lambda_{n, r} T\left(L_{n, r} ; 2\right) & =\int_{0}^{\pi} \frac{\left(\sin n \frac{\theta}{2}\right)^{2 r}}{\left(\sin \frac{\theta}{2}\right)^{2 r-2 \alpha-3}}\left(\cos \frac{\theta}{2}\right)^{2 \beta+1} d \theta \\
& \leq \pi^{2 r-2 \alpha-3}\left(\frac{n}{2}\right)^{2 r} \int_{0}^{\pi / n} \theta^{2 \alpha+3} d \theta+\pi^{2 r-2 \alpha-3} \int_{\pi / n}^{\pi} \theta^{2 \alpha+3-2 r} d \theta \\
& \leq\left(\frac{\pi}{2}\right)^{2 r+1} \frac{n^{2 r-2 \alpha-4}}{\alpha+2}+\frac{n^{2 r-2 \alpha-4}}{2 r-2 \alpha-4} \pi \\
& =n^{2 r-2 \alpha-4}\left(\left(\frac{\pi}{2}\right)^{2 r+1} \frac{1}{\alpha+2}+\frac{\pi}{2 r-2 \alpha-4}\right) .
\end{aligned}
$$

On the other hand $(n \geq 2)$

$$
\begin{aligned}
\lambda_{n, r} \int_{0}^{\pi / n} \frac{\left(\sin n \frac{\theta}{2}\right)^{r}}{\left(\sin \frac{\theta}{2}\right)^{2 r-2 \alpha-3}}\left(\cos \frac{\theta}{2}\right)^{2 \beta+1} d \theta & \geq\left(\frac{n}{\pi}\right)^{2 r} 2^{2 r-2 \alpha-3-\beta-1 / 2} \int_{0}^{\pi / n} \theta^{2 \alpha+3} d \theta \\
& =\left(\frac{n}{\pi}\right)^{2 r-2 \alpha-4} \frac{2^{2 r-2 \alpha-4-\beta-1 / 2}}{(\alpha+2)} .
\end{aligned}
$$

If we choose $\varepsilon>0$, then for $n>\pi / \varepsilon$

$$
\int_{0}^{\varepsilon} L_{n, r}(\theta) \rho^{(\alpha, \beta)}(\theta) d \theta \geq C_{B} \int_{0}^{\pi} L_{n, r}(\theta) \rho^{(\alpha, \beta)}(\theta) d \theta,
$$

where

$$
C_{B}=\frac{2^{2 r-2 \alpha-4-\beta-1 / 2}}{\pi^{2 r-2 \alpha-4}(\alpha+2)}\left(\left(\frac{\pi}{2}\right)^{2 r+1} \frac{1}{\alpha+2}+\frac{\pi}{2 r-2 \alpha-4}\right)^{-1} .
$$

Since $T\left(L_{n, r} ; 2\right) \approx n^{-2}$ it follows from $(2.9)$ and theorem 1.5 that the kernel $L_{n, r}(\theta)$ is saturated with order $n^{-2}$ and that the saturation class is $\operatorname{Lip}(2, X)$.

5.3. The Weierstrass kernel. The Weierstrass kernel, defined by

$$
W_{t}(\cos \theta)=\sum_{k=0}^{\infty} e^{-k(k+\alpha+\beta+1) t} \omega_{k}^{(\alpha, \beta)} R_{k}^{(\alpha, \beta)}(\cos \theta)
$$

is a positive kernel (see Bavinck [2], section 5.8). If we take a sequence of numbers $\left\{t_{n}\right\}$ with $\lim _{n \rightarrow \infty} t_{n}=0$, then it is easy to show that the sequence of convolution operators $W_{t_{n}}$ satisfies condition $(a)$ of theorem 2.3. In fact

$$
\lim _{t_{n} \rightarrow 0+} \frac{1-e^{-k(k+\alpha+\beta+1) t_{n}}}{1-e^{-(\alpha+\beta+2) t_{n}}}=\frac{k(k+\alpha+\beta+1)}{\alpha+\beta+2} .
$$


Hence by theorem 1.5 the sequence $W_{t_{n}}$ is saturated with order

$$
1-e^{-(\alpha+\beta+2) t_{n}} \approx t_{n} \quad(n \rightarrow \infty)
$$

and the saturation class in $\operatorname{Lip}(2, X)$.

\section{REFERENCES}

[1] R. A. ASKey AND S. WAINGER, A convolution structure for Jacobi series, Amer. J. Math., 91 (1969), 463-485.

[2] H. Bavinck, Approximation processes for Fourier-Jacobi expansions, Math. Centrum Amsterdam, report TW 126 (1971).

[ 3 ] H. BERENS, Interpolationsmethoden zur Behandlung von Approximationsprozessen auf Banachräumen, Lecture Notes in Math. 64, Springer, Berlin 1968.

[4] R. A. DeVore, On a saturation theorem of Tureckii, Tôhoku Math. J. 23 (1971), 353-362.

[5] G. Gasper, Positivity and the convolution structure for Jacobi series, Ann. of Math., 93 (1971), 112-118.

[6] E. GöRlich UND E. L. STARK, Ueber beste Konstanten und asymptotische Entwicklungen positiver Faltungsintegrale und deren Zusammenhang mit dem Saturationsproblem. Jber. Deutsch. Math. - Verein 72 (1970), 18-61.

[7] J. LöfSTRöm and J. PeEtre, Approximation theorems connected with generalized translations, Math. Ann., 181 (1969), 255-268.

[8] E. L. StARK, Ueber trigonometrische singuläre Faltungsintegrale mit Kernen endlicher Oszillation, Dissertation, Aachen (1970).

[9] G. Szegö, Orthogonal polynomials, Amer. Math. Soc. Coll. Publ., 23 (1967), Providence, R. I.

[10] A. H. TURECKII, On classes of saturation for certain methods of summation of Fourier series, Amer. Math. Soc. Trans., (2) 26 (1963), 268-272. (Uspehi Mat. Nauk 15 (1960) no. 6 (96), 149-156).

Mathematisch Centrum

AMSTERDAM, NEDERLAND 
